J. Lake Sci. (湖泊科学), 2014, 26(6): 864-870

http: //www. jlakes.org. E-mail : jlakes@niglas.ac.cn

(C) 2014 by Journal of Lake Sciences

\title{
温度对河蚬 (Corbicula fluminea) 氮、磷排泄影响的初步研究”
}

\author{
薛庆举 ${ }^{1,2}$, 蔡永久 $^{1}$, 许 浩 $^{1,2}$, 龚志军 $^{1 * *}$ \\ ( 1 : 中国科学院南京地理与湖泊研究所湖泊与环境国家重点实验室, 南京 210008) \\ (2: 中国科学院大学,北京 100049)
}

\begin{abstract}
摘 要: 在不同温度下, 对太湖河蚬进行了室内短期氮、磷释放模拟实验. 结果表明,温度对河蚬 (Corbicula fluminea) 不同 形态氮、磷的排泄都有重要的影响. 总磷 $(\mathrm{TP})$ 、总氮 $(\mathrm{TN})$ 、总溶解性氮 ( TDN)、总溶解性磷 $(\mathrm{TDP})$ 和正磷酸盐 $\left(\mathrm{PO}_{4}^{3-}-\mathrm{P}\right)$ 排泄率随温度的升高都显著升高, 而亚硝态氮 $\left(\mathrm{NO}_{2}^{-}-\mathrm{N}\right)$ 排泄率先升高后下降. 铵态氮 $\left(\mathrm{NH}_{4}^{+}-\mathrm{N}\right)$ 排泄率在 5 和 $15^{\circ} \mathrm{C}$ 间变 化不显著, 温度对硝态氮 $\left(\mathrm{NO}_{3}^{-}-\mathrm{N}\right)$ 排泄率有影响但不显著. 同时实验发现河蚬为排氨动物, $\mathrm{NH}_{4}^{+}-\mathrm{N}$ 排泄占 $\mathrm{TN}$ 排泄的 $50.78 \% \sim 100 \%$, TDN 排泄占 TN 排泄的 $89.14 \% \sim 100 \%$, 而 $\mathrm{NO}_{3}^{-}-\mathrm{N} 、 \mathrm{NO}_{2}^{-}-\mathrm{N}$ 虽都有检出, 但所占比例相对较小. 在河蚬 磷排泄中, TDP 占 TP 比例范围为 $83.01 \% \sim 100 \%, \mathrm{PO}_{4}^{3-}-\mathrm{P}$ 在 $36.60 \% \sim 96.59 \%$ 之间, 且所占比例都有随温度升高而增 加的趋势. 同时对排泄率与干重和温度之间的关系进行了回归分析, 发现不同氮、磷排泄率 $\left(\mathrm{NO}_{3}^{-}-\mathrm{N}^{-}\right.$和 $\mathrm{NO}_{2}^{-}-\mathrm{N}$ 除外) 与温度 和干重的关系均符合方程 $R(X)=a W^{b} \cdot \mathrm{e}^{c T}+d$, 决定系数 $R^{2}$ 都在 0.967 以上.
\end{abstract}

关键词: 太湖; 河蚬; 氮、磷排泄; 温度; 比重

\section{Effects of temperature on the nitrogen and phosphorus excretion of Corbicula fluminea}

\author{
XUE Qingju $^{1,2}$, CAI Yongjiu ${ }^{1}$, XU Hao ${ }^{1,2}$ \& GONG Zhijun ${ }^{1}$ \\ (1: State Key Laboratory of Lake Science and Environment, Nanjing Institute of Geography and Limnology, Chinese Academy \\ of Sciences, Nanjing 210008, P. R. China) \\ (2: University of Chinese Academy of Sciences, Beijing 100049, P. R. China)
}

\begin{abstract}
A microcosm was established to simulate the short-term nutrient excretion of the Corbicula fluminea which was sampled in the Gonghu Bay, Lake Taihu. The C. fluminea was cultivated at 5,15 and $25^{\circ} \mathrm{C}$ after 24 hours acclimation. The results showed that temperature greatly affected the nitrogen and phosphorus excretion rate of the $C$. fluminea. The excretion rate of total phosphorus (TP), total nitrogen( TN), total dissolved nitrogen(TDN), total dissolved phosphorus(TDP) and phosphate phosphorus $\left(\mathrm{PO}_{4}^{3-}-\mathrm{P}\right)$ represented a notable increase with the temperature increasing, however, the excretion rate of nitrite nitrogen $\left(\mathrm{NO}_{2}^{-}-\mathrm{N}\right)$ increased first and then reduced with the increasing temperature. The change in the excretion of ammonium nitrogen $\left(\mathrm{NH}_{4}^{+}-\mathrm{N}\right)$ between 5 and $15^{\circ} \mathrm{C}$ was not significant. Temperature had a non-significant effect on nitrate nitrogen $\left(\mathrm{NO}_{3}^{-}-\mathrm{N}\right)$. We also found that the $C$. fluminea was ammonotelic benthos, and $\mathrm{NH}_{4}^{+}-\mathrm{N}$ was the main nitrogenous excretory product which occupied for $50.78 \%-$ $100 \%$ of the TN. TDN accounted for $89.14 \%-100 \%$ of the TN. Although $\mathrm{NO}_{3}^{-}-\mathrm{N}$ and $\mathrm{NO}_{2}^{-}-\mathrm{N}$ were detected, the amount of them is very little. In the phosphorus excretion, the proportion of TDP and $\mathrm{PO}_{4}^{3-}-\mathrm{P}$ to $\mathrm{TP}$ were $83.01 \%-100 \%$ and $36.60 \%-$ $96.59 \%$, respectively, and the proportion of them increased with the rising temperature. Regression analysis between the dry weight, temperature and the excretion rates indicated that the regression equation $R(X)=a W^{b} \cdot \mathrm{e}^{c T}+d$ was adaptive to all types of the nitrogen and phosphorus excretion except $\mathrm{NO}_{3}^{-}-\mathrm{N}$ and $\mathrm{NO}_{2}^{-}-\mathrm{N}$, and the $R$ square reached over 0.967 .
\end{abstract}

Keywords: Lake Taihu; Corbicula fluminea; nitrogen and phosphorus excretion; temperature; proportion

* 国家自然科学基金项目 $(41230744,31270505)$ 和中国科学院南京地理与湖泊研究所 “一三五” 战略发展规划项目 ( NIGLAS2012135002) 联合资助. 2013-11-29 收稿;2013-12-30 收修改稿. 薛庆举 (1987 ), 男, 博士研究生; E-mail:qingju871228@163.com.

** 通信作者;E-mail:zjgong@ niglas. ac.cn. 
河蚬 (Corbicula fluminea) 属于瓣鳃纲、真瓣鳃目、蚬科, 最大壳长 $3.5 \mathrm{~cm}$, 外形略成正三角形, 壳表具同 心生长纹, 营穴居生活, 喜栖息于沙底或泥沙底的河流及湖泊内, 适宜生长的水温为 $9 \sim 32^{\circ} \mathrm{C}^{[1-2]}$. 河蚬原产 于东亚 (中国、泰国、菲律宾、朝鲜半岛、日本、俄罗斯东部等)、大洋洲、非洲,目前已经人侵到北美洲、南美洲 和欧洲等地, 现已广泛分布于世界各地的水域, 并对当地水域生态系统的物质循环和能量流动产生了重要 影响 ${ }^{[34]}$. 河蚬作为许多江河湖泊底栖动物的优势种, 不但在水产养殖中有重要的应用 ${ }^{[5]}$, 在水体营养盐循 环中也具有重要的作用.

国外关于底栖动物营养盐释放的研究可追溯到 20 世纪初, 而对双壳贝类比较系统的研究开始于 $1970 \mathrm{~s}^{[6-7]}$. 大部分研究结果表明底栖动物在湖泊营养盐循环中具有重要的作用 ${ }^{[8-11]}$. 一方面底栖动物可以通 过排泄和摄食等作用直接影响水体中营养盐的含量, 如 Tatrai ${ }^{[12]}$ 发现有底栖动物存在的实验组中铵态氮释 放量较无底栖动物组的高 $5 \sim 20$ 倍, 而 Devine 等 ${ }^{[13]}$ 对一个富营养水库的研究也发现, 底栖动物排泄的铵态 氮量接近于由流域进人湖泊的量,达到水库底泥释放总量的 $55 \%$, 反应性磷也可达到底泥释放总量的 $1 / 4$ 左右. 另一方面底栖动物还可以通过影响沉积物一水界面的环境条件和界面微生物的数量和活性, 从而间接 影响界面营养盐的交换 ${ }^{[1-16]}$.

国内对底栖动物营养盐释放的研究起步较晚, 且研究对象主要为海洋底栖动物 ${ }^{[17-20]}$, 而对淡水底栖动 物的研究在近几年才逐渐得到重视 ${ }^{[21-23]}$. 河蚬作为底栖动物的重要组成部分, 国内外对它的研究大多集中 于生理生态学和人侵危害等方面 ${ }^{[3]}$, 而它在湖泊营养盐循环中的重要作用一直没有得到足够重视. 河蚬是 太湖的主要优势种之一, 同时还是太湖仅次于银鱼的第二大创汇水产资源 ${ }^{[24]}$, 在湖中有广泛的分布. 同时, 相关研究表明,浮游植物和水生植物等水生生物对不同形态营养盐的吸收具有一定的选择性 ${ }^{[25-26]}$, 所以开 展河蚬营养盐代谢的研究不但可以明确河蚬在湖泊生态系统功能中的地位, 还可以加深对太湖氮、磷循环 过程以及蓝藻水华形成机理的认识. 本研究于 2012 年 11 月对太湖贡湖湾内的河蚬进行了室内短期营养盐 释放实验,得到河蚬在不同温度下氮、磷的释放速率, 同时计算出各形态氮、磷在总氮、总磷中所占的比例, 并且通过回归分析得到排泄率与河蚬干重以及温度的关系方程, 为进一步研究河蚬在湖泊营养盐循环中的 作用提供一些基础资料.

\section{1 材料与方法}

\section{1 研究地点概况}

基于先前对太湖河蚬时空分布格局的研究 ${ }^{[3]}$, 将采样点设在太湖东北部的贡湖湾, 位置坐标为 $\left(31^{\circ} 23^{\prime} 14^{\prime \prime} \mathrm{N}, 120^{\circ} 17^{\prime} 44^{\prime \prime} \mathrm{E}\right)$. 贡湖湾原为沉水植物控制的草型湖区 ${ }^{[27]}$, 且湖区内水体流动性好、富氧程度 高, 水质较好 ${ }^{[28]}$, 是无锡和苏州两市重要的水源地,但是随着近年来贡湖湾水体富营养化加剧, 野外调查 观测显示, 其北部水草区内沉水植物群落正逐渐消失 ${ }^{[29]}$, 而在本实验采样点处亦只有零星水草分布. 使用 自制三角拖网和 $1 / 40 \mathrm{~m}^{2}$ 改良 Peterson 采泥器采集河蚬和沉积物样品, 同时采集大约 $10 \mathrm{~L}$ 水样.

\section{2 实验设计}

将采集的河蚬迅速带回实验室, 挑选其中大小相似 (壳长在 $2.0 \mathrm{~cm}$ 左右) 且生命力旺盛的个体进行 $24 \mathrm{~h}$ 模拟驯化, 驯化中使用在采样点处采集的沉积物和水样. 驯化箱 (高 $\times$ 宽 $\times$ 长 $=22 \mathrm{~cm} \times 28 \mathrm{~cm} \times 41 \mathrm{~cm}$ ) 中沉 积物 (用篮网去除其中的河蚬) 厚 $3 \sim 5 \mathrm{~cm}$, 加水至箱体 $1 / 2$ 处, 驯化期间进行充分的曝气. 驯化结束后, 将河 蚬取出, 并用软毛刷和纯水清洗干净. 然后每 4 个 1 组 (根据采样区域冬季河蚬平均密度确定) 放人事先加 人 $700 \mathrm{ml}$ 纯水的 $1 \mathrm{~L}$ 高型烧杯 (直径 $9.5 \mathrm{~cm}$ ) 内, 再将烧杯放人已设置好温度的 GSX-300BS 型光照培养箱中 (光照度设为 $0 \%$ ). 实验设置 $5 、 15$ 和 $25^{\circ} \mathrm{C} 3$ 个温度梯度, 每个温度设 5 个平行和 1 个空白对照, 培养时间为 $24 \mathrm{~h}$. 分别在实验开始 $0 、 8 、 16$ 和 $24 \mathrm{~h}$ 时采集 $100 \mathrm{ml}$ 水样进行分析.

\section{3 参数测定}

水样分两部分进行测定,一部分直接用来检测总氮 (TN) 和总磷 (TP) , 另一部分经 Whatman GF/F 滤膜 过滤后检测总溶解性氮 $(\mathrm{TDN})$ 、总溶解性磷 $(\mathrm{TDP})$ 、铵态氮 $\left(\mathrm{NH}_{4}^{+}-\mathrm{N}\right)$ 、硝态氮 $\left(\mathrm{NO}_{3}^{-}-\mathrm{N}\right)$ 、亚硝态氮 $\left(\mathrm{NO}_{2}^{-}-\mathrm{N}\right)$ 和正磷酸盐 $\left(\mathrm{PO}_{4}^{3-}-\mathrm{P}\right)$. TN 、 TP、TDN 和 TDP 均采用过硫酸钾消解法测定, $\mathrm{NH}_{4}^{+}-\mathrm{N} 、 \mathrm{NO}_{3}^{-}-\mathrm{N} 、 \mathrm{NO}_{2}^{-}-\mathrm{N}$ 和 $\mathrm{PO}_{4}^{3-}-\mathrm{P}$ 均直接使用荷兰 Skalar 公司产的连续流动分析仪测定,所有样品的分析都在当天完成. 
在实验结束后, 将河蚬在 $65^{\circ} \mathrm{C}$ 下烘干至恒重, 然后用电子天平 (精度为 $0.1 \mathrm{mg}$ ) 测其干重, 河蚬干重如 表 1 所示. 经方差分析,不同温度不同平行之间河蚬干重(去壳)不存在显著性差异 $(P>0.05)$.

表 1 不同温度下河蚬干重

Tab. 1 Dry weight of Corbicula fluminea at different temperatures

\begin{tabular}{cccccc}
\hline 温度 $/{ }^{\circ} \mathrm{C}$ & \multicolumn{5}{c}{ 干重 $/ \mathrm{g}$} \\
\hline 5 & $0.1567 \pm 0.0271$ & $0.2302 \pm 0.0622$ & $0.2467 \pm 0.0436$ & $0.1617 \pm 0.0395$ & $0.1913 \pm 0.0796$ \\
15 & $0.1555 \pm 0.0564$ & $0.2196 \pm 0.0776$ & $0.1905 \pm 0.0554$ & $0.2139 \pm 0.1035$ & $0.2865 \pm 0.1107$ \\
25 & $0.1966 \pm 0.0742$ & $0.1979 \pm 0.0854$ & $0.1815 \pm 0.0406$ & $0.1485 \pm 0.0618$ & $0.2273 \pm 0.0532$ \\
\hline
\end{tabular}

实验中由于烧杯中水的体积不断减少,所以按照下面公式对后面 3 次采样中各物质浓度进行转换:

$$
\begin{aligned}
& C_{2}^{*}=C_{1}+6 / 7\left(C_{2}-C_{1}\right) \\
& C_{3}^{*}=C_{2}^{*}+5 / 7\left(C_{3}-C_{2}\right) \\
& C_{4}^{*}=C_{3}^{*}+4 / 7\left(C_{4}-C_{3}\right)
\end{aligned}
$$

式中, $C^{*}$ 为各物质体积转换后的浓度, $C_{1} \sim C_{4}$ 为 4 次采样各物质的实际浓度.

排泄率按照以下公式进行计算:

$$
R(X)=\left(C_{t}-C_{0}\right) /(W \cdot t)
$$

式中, $R(X)$ 表示物质 $X$ 的排泄率, $C_{t}$ 表示物质 $X$ 在 $t$ 时转换后的浓度, $C_{0}$ 表示物质 $X$ 在实验开始时的浓度, $W$ 为每个平行样河蚬的干重, $t$ 表示采样时的培养时间.

使用 SPSS 20.0 进行回归分析和方差分析, 采用 Origin 9.0 绘图.

\section{2 结果与分析}

\section{1 不同形态氮、磷的排泄率}

河蚬不同形态氮、磷排泄率如图 1 所示, 可以看出, 在 $5^{\circ} \mathrm{C}$ 时, $\mathrm{TN}$ 和 $\mathrm{TP}$ 排泄率随时间的延长都先降低后 略升高, 而 $\mathrm{PO}_{4}^{3-}-\mathrm{P}$ 先升高后降低, 其它物质排泄率皆随时间的延长表现出不同程度的下降; $15^{\circ} \mathrm{C}$ 时各物质 的排泄率都有逐渐下降的趋势; 而在 $25^{\circ} \mathrm{C}$ 时, TP 和 TDP 排泄率先升高后降低, $\mathrm{NO}_{3}^{-}-\mathrm{N}$ 排泄率不断升高, 其 它物质排泄率却不断下降.

单因素方差分析表明, 温度对不同形态氮、磷排泄率的影响除 $\mathrm{NO}_{3}^{-}-\mathrm{N}$ 以外都达到显著或极显著水平, 其中, $\mathrm{NH}_{4}^{+}-\mathrm{N}$ 排泄率在 5 和 $15^{\circ} \mathrm{C}$ 下变化不显著. 只有 $\mathrm{NO}_{2}^{-}-\mathrm{N}$ 排泄率随温度升高先升高后降低, 其它都随温 度升高而不断升高.

\section{2 不同形态氮、磷排泄量在 TN、TP 排泄量中的比例}

在河蚬的氮排泄中, $\mathrm{TDN} / \mathrm{TN}$ 在 $5^{\circ} \mathrm{C}$ 时为 $95.65 \% \sim 100 \%$, 在 $15^{\circ} \mathrm{C}$ 时为 $89.14 \% \sim 100 \%, 25^{\circ} \mathrm{C}$ 时在 $89.52 \% \sim 92.75 \%$ 之间随时间延长呈降低的趋势; $\mathrm{NH}_{4}^{+}-\mathrm{N} / \mathrm{TN}$ 除在 $5^{\circ} \mathrm{C}$ 时呈下降趋势之外, 在 15 和 $25^{\circ} \mathrm{C}$ 时 都是先上升后下降, 比例在 50.78\% 100\% 之间; $\mathrm{NO}_{3}^{-}-\mathrm{N}$ 和 $\mathrm{NO}_{2}^{-}-\mathrm{N}$ 在 TN 中所占的比例由于受环境条件的 影响较大,在不同的温度下表现出不同的变化趋势, 它们的比例分别在 $0.14 \% \sim 3.91 \%$ 和 $0.57 \% \sim 3.00 \%$ 之间, 但是都有随温度升高而不断下降的趋势 ( 图 2).

河蚬的磷排泄中, TDP 在 TP 中所占的比例在 $83.01 \% \sim 100 \%$ 之间, 有随温度升高而缓慢上升的趋势, 且随时间的延长最后都会出现降低; 而 $\mathrm{PO}_{4}^{3-}-\mathrm{P}$ 在 $\mathrm{TP}$ 中所占的比例除在 $5^{\circ} \mathrm{C}$ 开始有一低值 $(36.60 \%)$ 之外, 其它都在 $59.33 \% \sim 96.59 \%$ 之间, 温度高时比例相对较高 (图 2).

\section{3 排泄率与温度和干重之间的关系}

通过对不同方程模型进行回归分析, 结果显示回归方程都符合模型 $R(X)=a W^{b} \cdot \mathrm{e}^{c T}+d$, 且都达到显 著水平, 由于 $\mathrm{NO}_{3}^{-}-\mathrm{N}$ 与 $\mathrm{NO}_{2}^{-}-\mathrm{N}$ 受环境影响较大, 所以未列出分析结果. 各物质排泄率与干重皆为幂函数关 

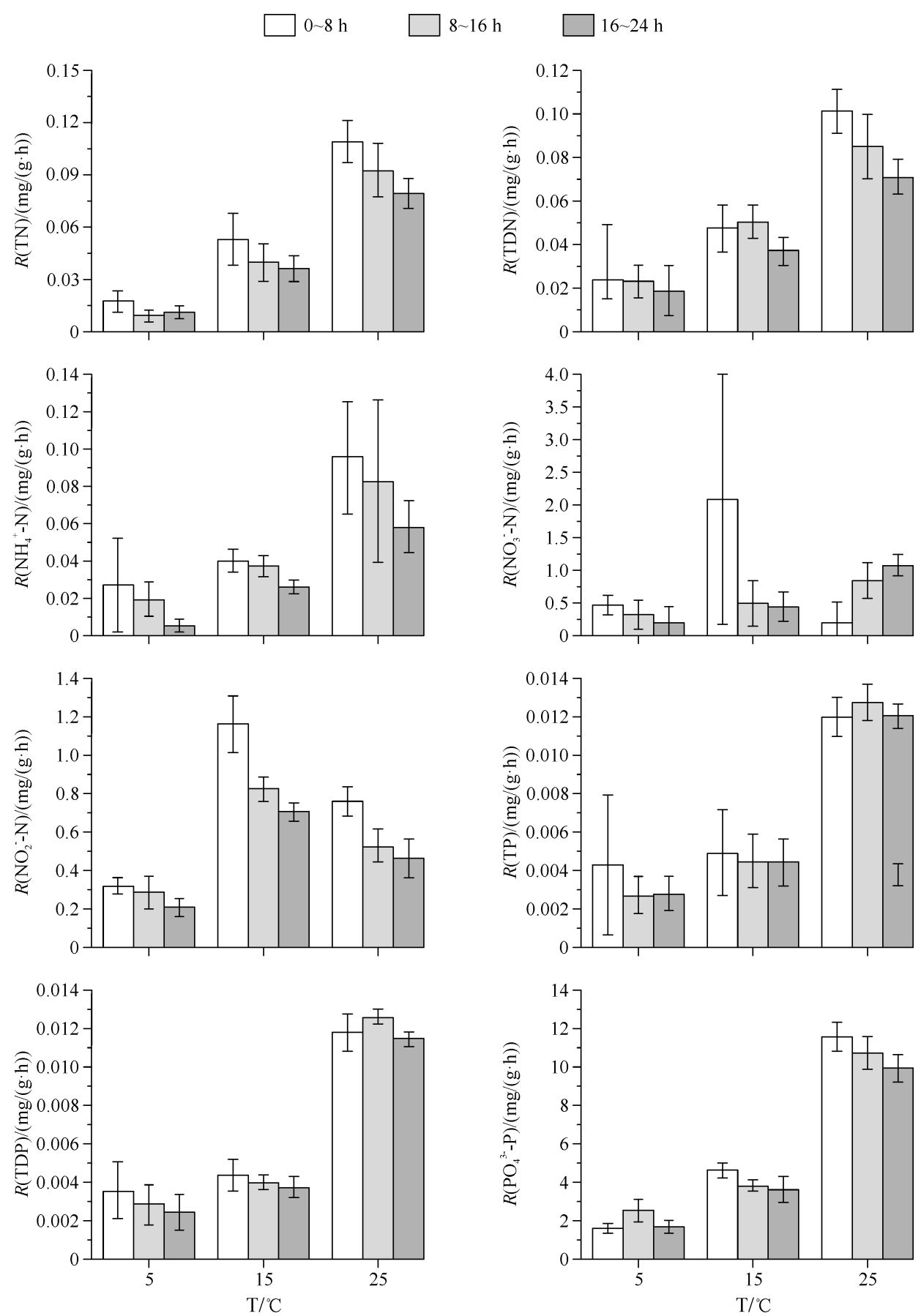

图 1 不同形态氮、磷排泄率在不同温度下随时间的变化

Fig. 1 Changes of excretion rate of the nitrogen and phosphate at different temperatures with time

系, 而与温度都为以 $\mathrm{e}$ 为底的指数函数关系. 其中 $a$ 值在 $0.0002 \sim 8.6227$ 之间, $b$ 值在 $-1.156 \sim-0.352$ 之 间, $c$ 值在 $0.0423 \sim 0.1692$ 之间, 而 $d$ 值在 $-0.0269 \sim 0.5513$ 之间, 各方程决定系数 $R^{2}$ 皆在 0.967 以上 (表 2). 结果表明, $\mathrm{TDN}$ 和 $\mathrm{NH}_{4}^{+}-\mathrm{N}$ 的排泄率与干重 $(\mathrm{W})$ 的关系相对更加显著, 而 $\mathrm{TP} 、 \mathrm{TDP}$ 和 $\mathrm{PO}_{4}^{3-}-\mathrm{P}$ 的排泄率与 温度 $(\mathrm{T})$ 的关系相对更加显著. 


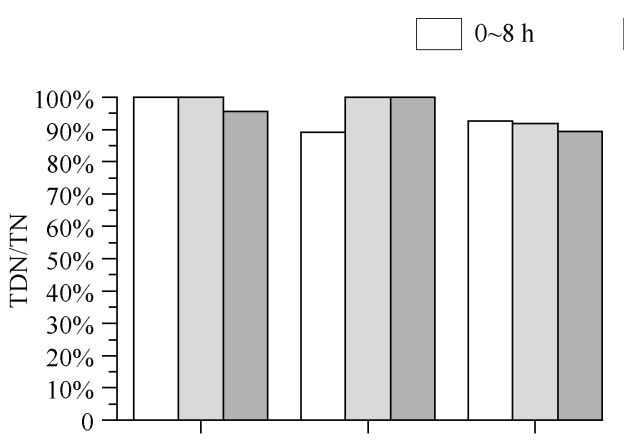

$8 \sim 16 \mathrm{~h} \square 16 \sim 24 \mathrm{~h}$
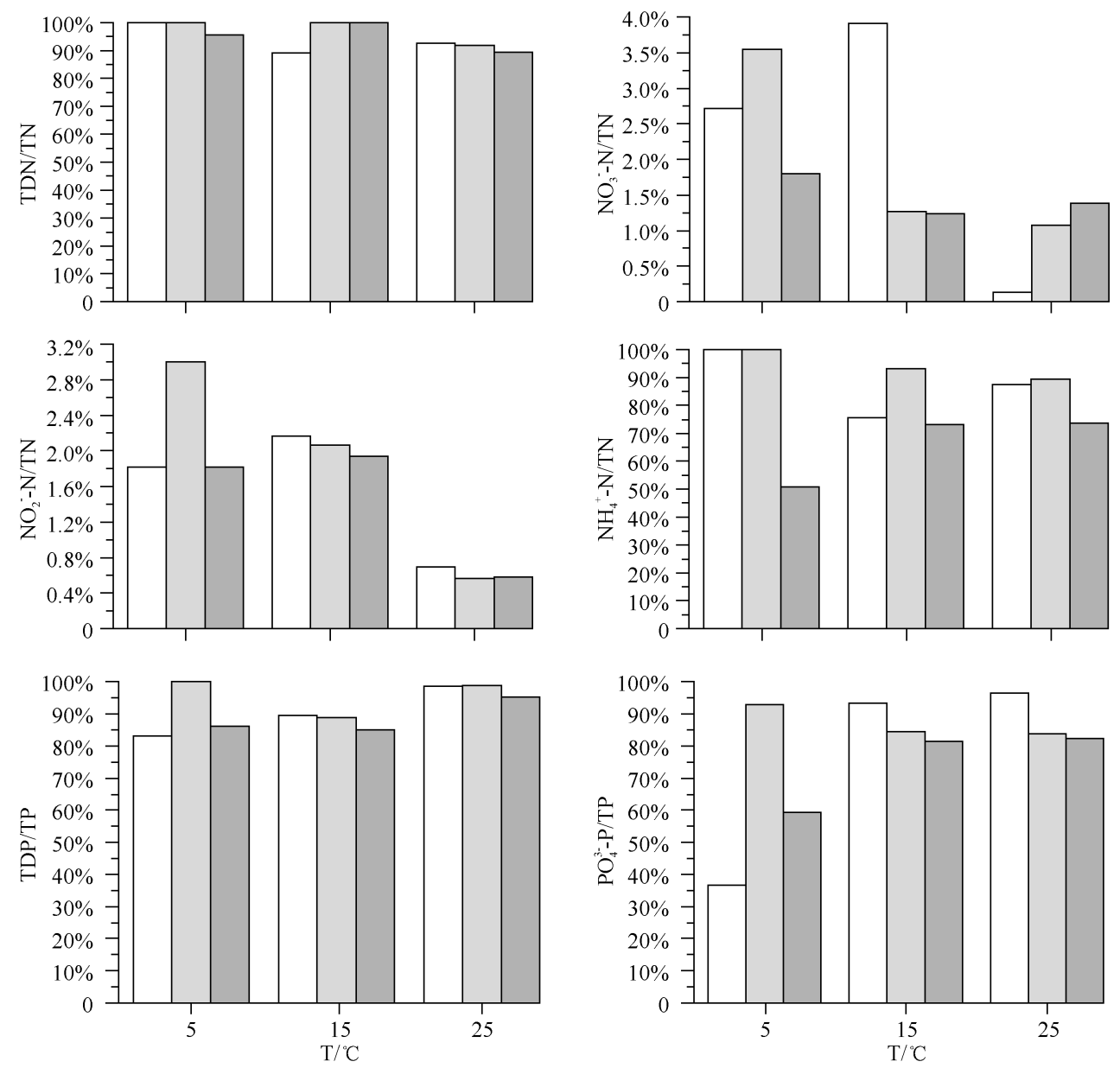

图 2 不同形态氮、磷比例在不同温度下随时间的变化

Fig. 2 Changes of the proportion of different types of nitrogen and phosphate with time

表 2 不同物质的排泄率与温度和干重的关系方程

Tab. 2 The regression equation between excretion rates and temperature, dry weight

\begin{tabular}{cccc}
\hline 水质指标 & 方程 & $R^{2}$ & $F$ \\
\hline $\mathrm{TN}$ & $R(\mathrm{TN})=0.0259 W^{-0.352} \cdot \mathrm{e}^{0.0423 T}-0.0269$ & 0.984 & 797.93 \\
$\mathrm{TDN}$ & $R(\mathrm{TDN})=0.0062 W^{-1.156} \cdot \mathrm{e}^{0.0726 T}+0.0003$ & 0.978 & 306.57 \\
$\mathrm{NH}_{4}^{+}-\mathrm{N}$ & $R\left(\mathrm{NH}_{4}^{+}-\mathrm{N}\right)=0.0046 W^{-1.150} \cdot \mathrm{e}^{0.0767 T}-0.0023$ & 0.975 & 350.27 \\
$\mathrm{TP}$ & $R(\mathrm{TP})=0.0002 W^{-0.478} \cdot \mathrm{e}^{0.1355 T}+0.0014$ & 0.967 & 380.50 \\
$\mathrm{TDP}$ & $R(\mathrm{TDP})=8.6227 W^{-0.515} \cdot \mathrm{e}^{0.1692 T}+0.0015$ & 0.984 & 441.68 \\
$\mathrm{PO}_{4}^{3-}-\mathrm{P}$ & $R\left(\mathrm{PO}_{4}^{3-}-\mathrm{P}\right)=0.3284 W^{-0.646} \cdot \mathrm{e}^{0.1138 T}+0.5513$ & 0.988 & 592.32 \\
\hline
\end{tabular}

\section{3 讨论}

\section{1 河蚬氮、磷排泄与温度和干重的关系}

本研究所得的排泄率与温度和干重的关系方程 $R(X)=a W^{b} \cdot \mathrm{e}^{c T}+d$ 与王俊、姜祖辉、宋协法等 ${ }^{[20,30-31]}$ 的研究基本一致. 大量研究表明, 温度和体重对不同底栖动物氮、磷排泄率都有显著的影响 ${ }^{[32-34]}$. 姜祖辉 
等 ${ }^{[30]}$ 研究发现菲律宾蛤仔氮、磷排泄率与温度呈正相关关系, 而与壳长呈幂指数函数关系. 刘其根等 ${ }^{[35]}$ 拟 合得出河蚬不同生长时期排氨率与温度关系的二次方程. 本实验经过二次方程、幂函数等多种函数的拟合 发现, 方程 $R(X)=a W^{b} \cdot \mathrm{e}^{c T}+d$ 的相关性最高. 其中各形态氮、磷排泄率均与干重呈幂函数关系, 且随干重 的增加大部分物质单位质量排泄率降低,而与温度都呈正相关关系.

\section{2 河蚬不同形态氮、磷排泄所占比例的变化规律}

贝类氮的排泄物一般包括 $\mathrm{NH}_{4}^{+}-\mathrm{N}$ 、尿素、氨基酸及某些胺类化合物等,而 $\mathrm{NH}_{4}^{+}-\mathrm{N}$ 所占比例最大,其余部 分的含量因物种不同而不同, 在贝类氮的排泄物中 $\mathrm{NO}_{3}^{-}-\mathrm{N}$ 和 $\mathrm{NO}_{2}^{-}-\mathrm{N}$ 所占的比例一般很小甚至检测不 到 ${ }^{[15,17,19-20,33]}$. 本研究结果显示, TDN 所占 TN 的比例基本都在 $90 \%$ 以上,甚至达到 100\%. 国内外研究表明, 双壳贝类都为排铵类动物, $\mathrm{NH}_{4}^{+}-\mathrm{N}$ 排泄物所占比例最高, 如周毅等 ${ }^{[18]}$ 对几种海洋双壳贝类的研究发现, $\mathrm{NH}_{4}^{+}-\mathrm{N}$ 占 TDN 比例的平均值在 70.8\% 80.1\% 范围内, 而 Cockcroft ${ }^{[36]}$ 发现贻贝排泄 $\mathrm{NH}_{4}^{+}-\mathrm{N}$ 占 $\mathrm{TDN}$ 的比 例为 70\% 78\%, 本实验的结果与其基本一致. 对于 $\mathrm{NO}_{3}^{-}-\mathrm{N}$ 和 $\mathrm{NO}_{2}^{-}-\mathrm{N}$ 的研究相对较少, Cockeroft 对贻贝的 研究发现, $\mathrm{NO}_{3}^{-}-\mathrm{N}$ 仅占 TDN 的 $1 \%$ 以下, 且未检测到 $\mathrm{NO}_{2}^{-}-\mathrm{N}$, 同时也有部分研究检测出两类物质,但比例都比 较小 ${ }^{[17-18]}$, 本实验结论与之相似. 本研究结果发现, 河蚬磷排泄物主要为 $\mathrm{PO}_{4}^{3-}-\mathrm{P}$, 所占 $\mathrm{TP}$ 比例在 $36.60 \% \sim$ $96.59 \%$ 之间, 这与一些学者对其它底栖动物的研究结果基本一致 ${ }^{[3740]}$. 而颗粒磷所占比例相对较小, 基本 都在 $17 \%$ 以下.

\section{3 河蚬氮、磷排泄率在不同温度下的变化规律}

温度是影响底栖动物氮、磷排泄的主要因子之一 ${ }^{[7]}$, 从本研究可以看出, 河蚬各形态氮、磷排泄率在一 定的温度范围内都会随温度的升高而显著升高,但到达一定的温度又会降低,且排泄率降低时的“拐点温 度” 因物质形态的不同而有所不同, 如 $\mathrm{NO}_{2}^{-}-\mathrm{N}$ 排泄率在 $15 \sim 25^{\circ} \mathrm{C}$ 之间就开始下降, 而其它物质在 $5 \sim 25^{\circ} \mathrm{C}$ 之 内均呈持续升高趋势 (图 1). 相关研究表明, 不同底栖动物物种排泄率 “拐点温度” 也会有所不同, 如角蝶螺 ( Turbo cornutus) $\mathrm{NH}_{4}^{+}-\mathrm{N}$ 排泄率在 $27.4^{\circ} \mathrm{C}$ 时达到最大, 河蚬为 $28^{\circ} \mathrm{C}$ 左右, 而日本刺沙蚌 (Neanthes japonica) 为 $26^{\circ} \mathrm{C}$, 菲律宾蛤仔 (Venerupis philippinarum) 低于 $25^{\circ} \mathrm{C}$, 有的却高达 $30^{\circ} \mathrm{C}^{[17,21-23,32,35,41]}$. 有学者指出实验条件和 动物发育时期也会对 “拐点温度” 产生影响 ${ }^{[7,33]}$. 由于河蚬在低温时停止摄食且呼吸和代谢速率显著降 低 ${ }^{[42]}$,所以实验中河蚬在低温时的排泄率也非常低.

不同形态氮的排泄率随时间延长都会有不同程度的降低,但是 $\mathrm{NO}_{3}^{-}-\mathrm{N}$ 在 $25^{\circ} \mathrm{C}$ 时排泄率却不断升高 (图 1 ), 这可能是由于在温度较高时随着时间的增加 $\mathrm{NO}_{2}^{-}-\mathrm{N}$ 形态不稳定. 其中 $\mathrm{NO}_{3}^{-}-\mathrm{N}$ 和 $\mathrm{NO}_{2}^{-}-\mathrm{N}$ 的排泄率都非 常小, 大部分在 $0.8 \mu \mathrm{g} /(\mathrm{g} \cdot \mathrm{h})$ 以下, 而 $\mathrm{NH}_{4}^{+}-\mathrm{N}$ 的排泄率要高出很多, 基本在 $0.01 \mathrm{mg} /(\mathrm{g} \cdot \mathrm{h})$ 以上, 甚至达 到 $0.067 \mathrm{mg} /(\mathrm{g} \cdot \mathrm{h})($ 图 1).

研究结果表明,在一定温度范围内,随温度升高河蚬不同形态磷的排泄率都显著升高 (图 1), 这与一些 学者的研究结果基本一致,如蒋红等 ${ }^{[17]}$ 发现菲律宾蛤仔不同形态磷的排泄率随温度升高略有升高,而姜祖 辉等 ${ }^{[30]}$ 对菲律宾蛤仔的研究也发现其排磷率与温度呈正相关关系, 但亦有学者在研究对虾时发现相反的结 果 ${ }^{[43]}$, 这可能与不同实验对象的生理活动状态有关.

\section{4 参考文献}

[ 1 ] 常亚青. 贝类增养殖学. 北京: 中国农业出版社,2007:140.

[2] 刘月英,张文珍,王跃先. 中国经济动物志一一淡水软体动物. 北京:科学出版社,1979:119-123.

[3] 蔡 炜,蔡永久,龚志军等. 太湖河蚬时空格局. 湖泊科学,2010,22(5):714-722.

[ 4 ] Karatayev AY, Padilla DK, Minchin D et al. Changes in global economies and trade: the potential spread of exotic freshwater bivalves. Biological Invasions, 2007, 9(2): 161-180.

[ 5 ] Buttner JK. Corbicula as a biological filter and polyculture organism in catfish rearing ponds. The Progressive Fish-Culturist, 1986, 48(2) : 136-139.

[ 6 ] Regnault M. Nitrogen excretion in marine and freshwater crustacean. Biological Reviews, 1987, 62(1): 1-24.

[ 7 ] 刘 勇,施坤涛,张少华等. 双壳贝类呼吸代谢的研究进展. 南方水产, 2007,3(4):65-69.

[ 8 ] Downing JA. Assessment of secondary production: the first step. In: Downing JA, Rigler FH eds. A manual on methods for the assessment of secondary productivity in fresh waters. Oxford: Blackwell Scientific, 1971. 
[9] Benke AC. Concepts and patterns of invertebrate production in running waters. Verh Internat Verein Limnol, 1993 , 25: $15-38$.

[10 ] Lindegaard C. Classification of water-bodies and pollution. The Chironomidae: The biology and ecology of non-biting midges. London: Chapman \& Hall, 1995 : 385-404.

[11] Wetzel RG. Limnology: Third Edition. San Diego: Academic Press, 2001: 665-730.

[12] Tatrai I. Rates of ammonia release from sediments by chironomid larvae. Freshwater Biology, 1986, 16(1): 61-66.

[13] Devine JA, Vanni MJ. Spatial and seasonal variation in nutrient excretion by benthic invertebrates in a eutrophic reservoir. Freshwater Biology, 2002, 47: 1107-1121.

[14] Lewandowski J, Laskov C, Hupfer M. The relationship between Chironomus plumosus burrows and the spatial distribution of pore-water phosphate, iron and ammonium in lake sediments. Freshwater Biology, 2007, 52(2): 331-343.

[15] Peter S. Enhanced exoenzyme activities in sediments in the presence of deposit-feeding Chironomus riparius larvae. Freshwater Biology, 2007, 52(9): 1807-1819.

[16] van de Bund WJ. Food web relation of littoral macro- and meiobenthos[Dissertation]. Amsterdam: University of Amsterdam, 1994.

[17] 蒋 红, 崔 毅, 陈碧鹃. 乳山湾菲律宾蛤仔可溶性氮、磷排泄及其与温度的关系. 中国水产科学, 2006, 13 (2) : 237-242.

[18］周 毅, 杨红生, 何义朝等. 四十里湾几种双壳贝类及污损动物的氮、磷排泄及其生态效应. 海洋与湖沼, 2002,33 (4) :424-431.

[19] 周 毅, 毛玉泽, 杨红生. 四十里湾栉孔扇贝清滤率、摄食率和吸收效率的现场研究. 生态学报, 2002,22(9): $1455-1462$.

[20］王 俊,姜祖辉,唐启升. 栉孔扇贝耗氧率和排氨率的研究. 应用生态学报,2002,13(9):1157-1160.

[21］㚞甄姣, 吴常文,皇甫淑燕. 温度、盐度、pH 对角蝾螺排氨率的影响. 渔业现代化,2007,34(3):10-12.

[22] 温晓蔓, 孙陆宇, 禹 娜等. 温度和盐度对背角无齿蚌 (Anodonta woodiana) 代谢的影响. 复旦学报: 自然科学版, 2011,50(5) : 632-639.

[23] 孙陆宇, 温晓曼, 禹 娜等. 温度和盐度对中华圆田螺和铜锈环棱螺标准代谢的影响. 中国水产科学, 2012,19(2): 275-282.

[24] 曹文明,周 刚,盛建明等. 太湖河蚬资源现状及演变. 南京林业大学学报: 自然科学版,2000, ( S1):125-128.

[25] 杨 柳,章 铭,刘正文.太湖春季浮游植物群落对不同形态氮的吸收. 湖泊科学,2011,23(4):605-611.

[26] 古滨河,刘正文,李宽意等译. 湖沼学一内陆水生态系统. 北京: 高等教育出版社, 2011:285.

[27] Qin BQ. Taihu Lake, China-dynamics and environmental change. Dordrecht: Springer, 2008.

[28］范成新,季 江, 张文华等. 贡湖水质富营养化综合评价及初步预测. 海洋湖沼通报, 1997, (3) : 18-24.

[29] 朱广伟. 太湖富营养化现状及原因分析. 湖泊科学, 2008,20(1):21-26.

[30］姜祖辉,王 俊. 菲律宾蛤仔氮、磷代谢的初步研究. 青岛大学学报,1999,12(2):68-73.

[31］宋协法, 刘 鹏, 葛长字. 温度、盐度交互作用对凡纳滨对虾耗氧和氨氮、磷排泄的影响. 渔业现代化, 2009,36(2) : 1-6.

[32] 刘 勇,线薇薇. 温度对日本刺沙亘氮生长和氮收支的影响. 水产科学, 2010,29(6):311-316.

[33] 吴 斌,廖思明,兰国宝. 水生无脊椎动物氮排泄研究概述. 广西科学, 2008,15(1):92-96.

[34] 李松青,林小涛,李卓佳等. 摄食对凡纳滨对虾耗氧率和氮、磷排泄率的影响. 热带海洋学报,2006,25(2):44-48.

[35] 刘其根,沈和定,周洪琪等. 河蚬的耗氧率和排氨率.上海水产大学学报, 1999,8(4):298-303.

[36] Cockcroft AC. Nitrogen excretion by the surf zone bivalves Donax serra and D. sordidus. Marine Ecology Progress Series, 1990, 60: 57-65.

[37 ] 阮景荣. 三种鱼的磷排泄及其在微型生态系统磷再循环中的作用. 水生生物学报,2005,29(1) :55-60.

[38] Brabrand A, Faafeng BA, Nilssen JPM. Relative importance of phosphorus supply to phytoplankton production: fish excretion versus external loading. Canadian Journal of Fisheries and Aquatic Sciences, 1990, 47(2) : 364-372.

[39] Arakelova ES. Periphyton grazing and phosphorus excretion by mollusks. Russian Journal of Ecology, 2010, 41 (4) : 327 332.

[40] Vink S, Atkinson MJ. High dissolved C: P excretion ratios for large benthic marine invertebrates. Marine Ecology Progress Series, 1985, 21 : 191-195.

[41] Aldridge DW, Payne BS, Andrew C. Miller oxygen consumption, nitrogenous excretion, and filtration rates of Dreissena polymorpha at acclimation temperatures between 20 and $32^{\circ} \mathrm{C}$. Canadian Journal of Fisheries and Aquatic Sciences, 1995 , 52(8): 1761-1767.

[42] 刘 敏,熊邦喜. 河蚬的生态习性及其对重金属的富集作用. 安徽农业科学, 2008,36(1):221-224.

[43] 孙成波, 李 婷, 李义军等. 多因子对斑节对虾氮磷代谢的影响. 浙江海洋学院学报: 自然科学版, 2011,30(2): 132-136. 